Advances in Radio Science (2004) 2: 7-12

(c) Copernicus GmbH 2004

\title{
Generation of short electrical pulses based on bipolar transistors
}

\author{
M. Gerding, T. Musch, and B. Schiek \\ RF and Microwave Institute, Ruhr-Universitaet Bochum, Universitaetsstrasse 150, 44780 Bochum, Germany
}

\begin{abstract}
A system for the generation of short electrical pulses based on the minority carrier charge storage and the step recovery effect of bipolar transistors is presented.

Electrical pulses of about 90 ps up to 800 ps duration are generated with a maximum amplitude of approximately $7 \mathrm{~V}$

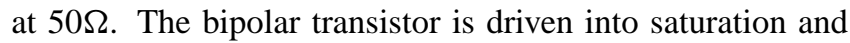
the base-collector and base-emitter junctions become forward biased. The resulting fast switch-off edge of the transistor's output signal is the basis for the pulse generation. The fast switching of the transistor occurs as a result of the minority carriers that have been injected and stored across the base-collector junction under forward bias conditions. If the saturated transistor is suddenly reverse biased the pn-junction will appear as a low impedance until the stored charge is depleted. Then the impedance will suddenly increase to its normal high value and the flow of current through the junction will turn to zero, abruptly.
\end{abstract}

A differentiation of the output signal of the transistor results in two short pulses with opposite polarities. The differentiating circuit is implemented by a transmission line network, which mainly acts as a high pass filter.

Both the transistor technology (pnp or npn) and the phase of the transfer function of the differentating circuit influence the polarity of the output pulses. The pulse duration depends on the transistor parameters as well as on the transfer function of the pulse shaping network.

This way of generating short electrical pulses is a new alternative for conventional comb generators based on steprecovery diodes (SRD). Due to the three-terminal structure of the transistor the isolation problem between the input and the output signal of the transistor network is drastically simplified. Furthermore the transistor is an active element in contrast to a SRD, so that its current gain can be used to minimize the power of the driving signal.

Correspondence to: M. Gerding

(michael.gerding@ruhr-uni-bochum.de)

\section{Introduction}

The use of rf-transistors as key components in comb generators provides a new, more comfortable and more cost effective way of generating short electrical pulses as compared to step-recovery diodes (HP, 1988). The achievable pulse length, the output power and pulse polarity can be influenced by the choice of the rf-transistor, the rf-transistor technology and the dimensions of the pulse shaping network. The transistor based circuit offers a wide range of different configurations so that its properties can be adapted to the field of applications relatively easily. The employed bipolar transistors are standard rf-components. This provides a good availability and a wide spectrum of different electronic specifications. The current gain of the transistor and its three terminal character are further advantages compared to a passive steprecovery diode. Because of this, low power input signals lead to an output peak pulse power of nearly $1 \mathrm{~W}$, e.g. $7 \mathrm{~V}$ at $50 \Omega$. The pulse duration can be adjusted between $90 \mathrm{ps}$ and $800 \mathrm{ps}$.

\section{Functional principle}

The two comb generators shown in Fig. 1 consist of a driver circuit, a slope accelerator or speed-up stage with a npn- or pnp-bipolar transistor and a pulse shaping network.

The key component of the speed-up stage is a rf-transistor which has to be driven into saturation in order to get an output signal with a fast switch-off slope, attributed to the minority carrier charge storage and the step recovery effect of the transistor (Paul, 1965). In fact the transistor's input signal has to fulfil some requirements, like a well defined amplitude and duration and a smooth rising leading edge. Therefore the driver circuit uses an edge-triggered input stage to provide a trigger signal to the speed-up network independent of the waveform, the duty-cycle and the amplitude of the comb generator's input signal. The output signal of the driver circuit is a comparably slow square wave pulse with a well defined width of a few nanoseconds. With the leading edge 


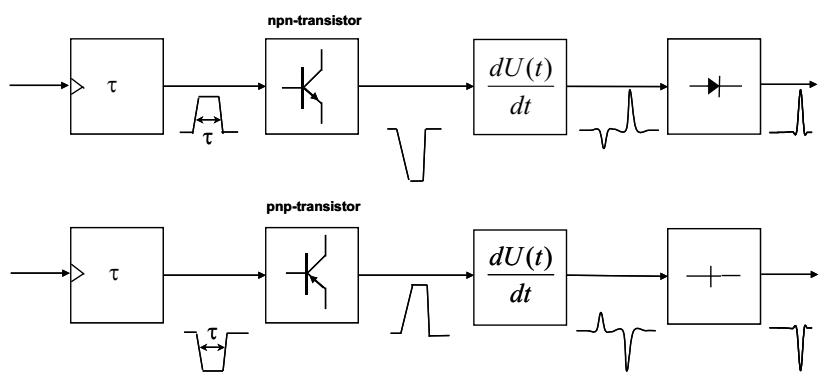

Fig. 1. Functional principle of the transistor based comb generator.

of the trigger signal, the base-emitter and the base-collector diode become forward biased: the transistor is in the saturation mode. The trailing edge of the trigger signal causes a fast depletion of the stored charge in the base-collector junction which leads to a sudden increase of the base-collector impedance. This causes an abrupt change of the voltage across the collector-emitter junction. Compared to the trailing edge of the input signal, the rise time at the output is shorter by a factor of approximately 10. By using fast logic gates for the driving stage of the speed up network, a resulting rise time of less than 100 ps can be achieved at the output of the transistor. The polarity of the fast switch-off edge can be influenced by the choice of the transistor technology and by the transistor circuit. The signals shown in Fig. 1 belong to a common emitter circuit. Because of its current gain the common emitter circuit proves to be very suitable for this application. The next element in the signal path is the so called pulse shaping network behind the transistor network. This circuit by itself can be divided into two parts. The first one, the differentiating network forms a pulse from the switch-off edge of the output signal of the transistor. A simple differentiation of the rectangular output signal leads to a signal, which shows two pulses with different pulse polarities and differently sharp pulses. Not only the fast trailing edge of the signal is being differentiated, the slower leading edge is differentiated, too. The comparably slow leading edge results in a low pulse amplitude, which has to be suppressed e.g. by a clipping network which can be based on a passive diode network or an active transistor network. Then the output signal of the comb generator will ideally contain a sharp and short pulse.

\section{Configuration of the comb generator}

As mentioned above, the comb generator consists of three different stages. Two of these are explained in detail in the following chapters.

\subsection{The speed-up network}

The speed-up network mainly consists of a transistor circuit. Although common base, common emitter or common collector circuits are all usable for this operation, this work is concentrated on the common emitter circuit, which is the most

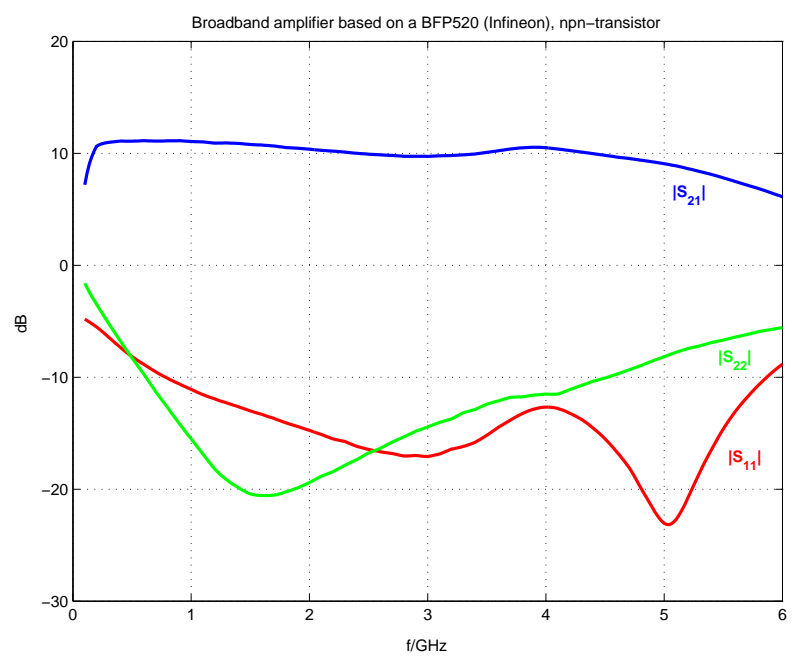

Fig. 2. Simulated Scattering parameters of a broadband amplifier based on a npn rf-transistor (BFP520, Infineon).

useful circuit for this application, because of its high current gain.

Short pulses with a pulse duration of about $100 \mathrm{ps}$ result in a broadband spectrum with a high corner frequency of several GHz. In a first step the transistor can be seen as a broadband amplifier realized as an emitter circuit. Therefore, the linear transient response of the transistor circuit is optimized for a flat gain of about $+10 \mathrm{~dB}$ and a flat frequency range up to $6 \mathrm{GHz}$. Figure 2 shows the simulated scattering parameters of the transistor circuit. Among others, the bandwidth of the amplifier depends on the specifications of the rf-transistor. As a rule of thumb, the faster the transistor, the shorter the switch-off edge.

As mentioned above, the transistor has to be driven into saturation, to get the fast step-recovery effect. Figure 3 shows a measurement of the input- and the output signal of a npntransistor in a common emitter circuit. As expected, the output signal (red line) is inverted, as caused by the common emitter circuit. Figure 3 gives a comparison of the falling edge of the input signal (blue line) and the corresponding rising edge of the output signal (red line).

\subsection{Differentiation network}

The differentiating network represents the first part of the pulse shaping network. In order to achieve pulses out of the rectangular output signal of the transistor network the output signal has to be differentiated. In general the transient response of the differentiating network shows a high-pass filter characteristic. The network can be implemented as a planar microstrip structure or as a transformer based structure. Both structures will be explained in detail in the following. The dimensions of the passive network influence the pulse shape, the pulse duration and its amplitude. Some layouts additionally offer the possibility of inverting the polarity of the signal resulting in an inversion of the output pulse. 


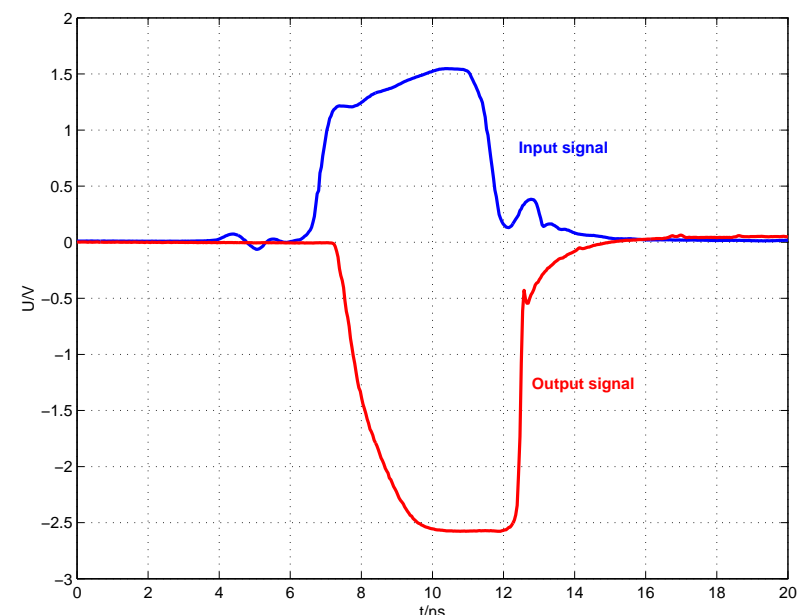

Fig. 3. Measurement of the input- and output signal of the speed-up network.

\subsubsection{Shorted branch line network}

Figure 4 shows the simplest realization of a planar differentiating network. The high-pass character which is shown as a simulation result in Fig. 4, is achieved by the branch line shorted to signal ground. Both, the width and the length of the branch line influence the pulse shape. The length of the line shifts the corner frequency of the transfer function and the width determines the line impedance. It is a disadvantage that there is no complementary structure to shift the phase of the input signal by $180^{\circ}$ in order to achieve a pulse inversion. The only way to get different pulse polarities by this structure is to use different transistor technologies, i.e. pnp-transistors instead of npn-transistors

\subsubsection{Coupled-line coupler as differentiating network}

The use of planar coupled-line structures solves the problem of the pulse inversion. The magnitude of the transfer function of the coupled-line structure is nearly equivalent to the shorted branch line structure mentioned above. In fact the coupled-line structure has two more parameters which have to be optimized. Aside from the length and the width of each line, the two additional parameters are the coupling between the lines and the termination of each line. As it is shown in Fig. 5 the lines can be left open or can be shorted to ground at their ends. It can be shown theoretically by the use of coupled-line equivalents and the Kuroda identities, that the different terminations result in a phase shifting of the input signal (Malherbe, 1979). The theoretical phase difference between the transfer function of both structures is $180^{\circ}$ at the quarter wavelength frequency and nearly $180^{\circ}$ over a wide frequency range. Because of this, the coupled-line structure with shorted lines changes the polarity of the output signal. When realizing these structures, the coupling between the two lines is critical. To maximize the coupling and to minimize the transmission loss, the coupling has to be tight. This means that the distance between the two lines has to be as

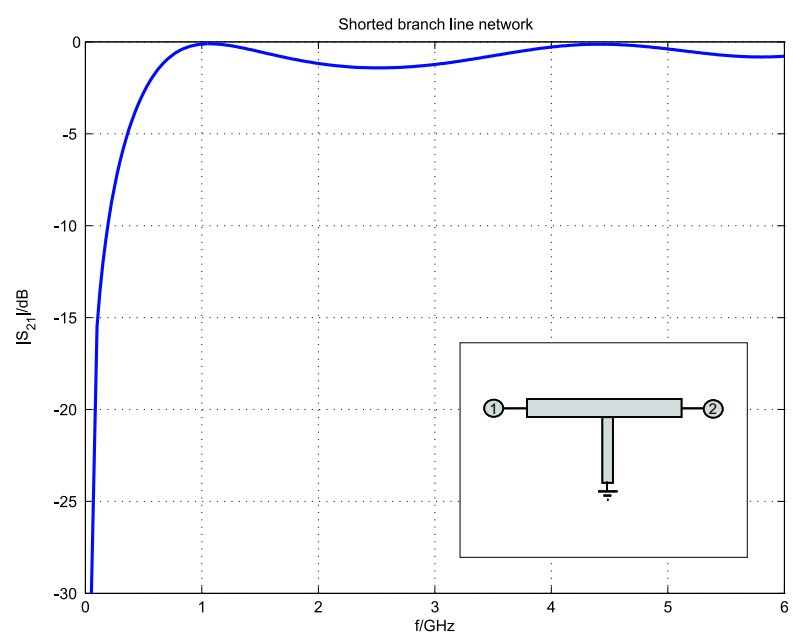

Fig. 4. Simulated transfer function of the shorted branch line network, optimized for relatively slow pulses.

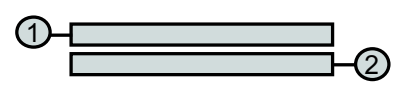

a)

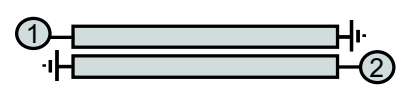

b)

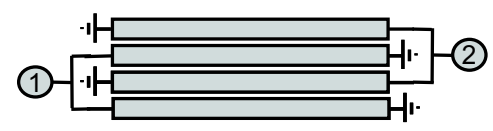

c)

Fig. 5. Coupled-line differentiating network: (a) not inverting, (b) and (c) inverting.

narrow as possible, e.g. $75 \mu \mathrm{m}$ or $100 \mu \mathrm{m}$. To overcome this mechanical limitation, two or more coupled-line structures can be combined in parallel.

Figure 6 illustrates the simulated results of the differential phase between the two alternative circuits (open end, shorted end). As expected, the difference is about $180^{\circ}$ over a wide frequency range.

\subsubsection{Transformer based differentiating network}

Instead of the planar structures, transformers can be used as differentiating networks. Their function is comparable to the coupled-line structures. This means, coupling, input and output impedance and the possibility of changing the pulse polarity are adjustable parameters. As it is illustrated in Fig. 7, the direction of winding influences the signal inversion.

The network cannot be used at very high frequencies, because the upper limiting frequency is determined by the ferrite and the structure of the windings. 

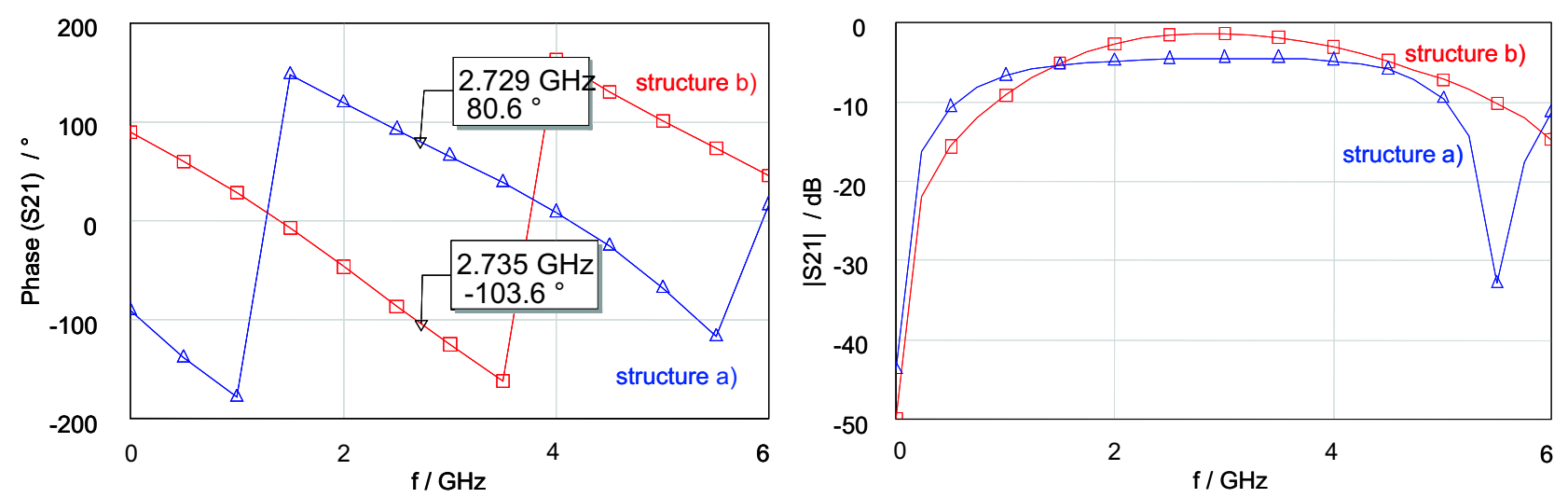

Fig. 6. Simulated phase and magnitude of the transfer function of different coupled-line structures (see Fig. 5), open end (a), shorted end (b).

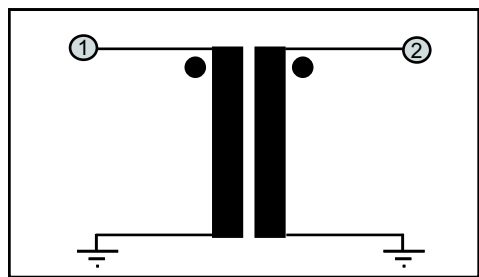

not inverting

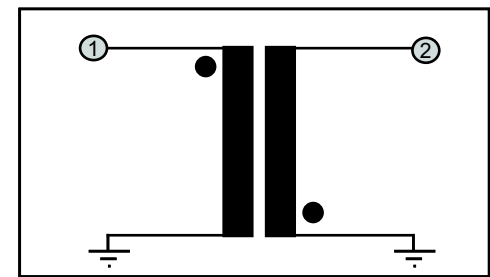

inverting

Fig. 7. Functional principle of transformer based differentiating networks (inverting, not inverting).

\subsection{Function of the clipping network}

The second stage of the pulse shaping network consists of the so called clipping network. Although there are several possible realisations of clipping networks, this contribution focuses on a passive, relatively simple and robust method. A fast Schottky diode is connected in series with the output of the differentiating stage. Depending on the diode's polarity, the negative or the positive part of the input signal is clipped.

Figure 8 illustrates this functionality. The left diagram shows the output signal of the differentiating stage at the input of the clipping network. The slow negative pulse, which is a result of the differentiation of the slow leading edge of the output signal of the transistor network, should be cancelled by the clipping network. The right diagram in Fig. 8 shows the output signal of the clipping network. The slow negative pulse is nearly completely eliminated as desired. The small residual signal is caused by a capacitive coupling which can mainly be explained by the junction capacitance and the parasitic capacitance of the diode. The smaller the parasitic capacitance the smaller the coupled rest of the signal. Furthermore, the diode should have a very low forward resistance to minimize the forward voltage drop across the diode.

\section{Results of exemplary measurements}

\subsection{Comparison of different pulse polarities}

Figure 9 gives an overview of different pulse shapes with different polarities and pulse durations. As mentioned above, the pulse polarity can be determined by the choice of the transistor technology (npn or pnp) and by the proper realization of the pulse shaping network. The left diagram shows two traces measured with two identical pulse generator circuits both with a shorted branch line as a differentiating network. They only differ in the transistor used. Comparing both output signals, the negative pulse is longer than the positive one by a factor of 1.5. Taking the transient frequency of a transistor as criteria, npn-transistors are generally faster, which results in shorter rise times of the switch-off edge and thus in shorter pulses.

The pulse inversion based on different coupled line structures is shown in the right part of Fig. 9. The same fast signal generation based on a npn-transistor can be used for both pulse polarities so that the pulse shape and the pulse length only depend on the coupling network. By using the coupledline structure for differentiating the pulse and for inverting the pulse, the pulse duration is roughly the same. 

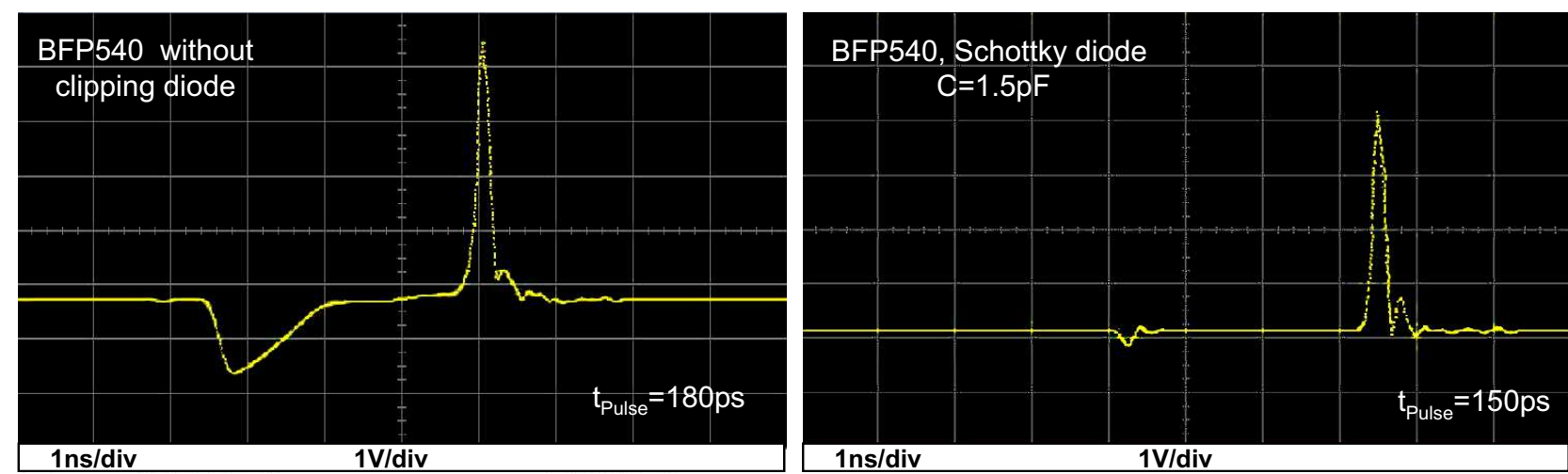

Fig. 8. Measured input and output signal of the clipping stage, left: input signal, right: output signal based on a standard Schottky diode as the clipping element.
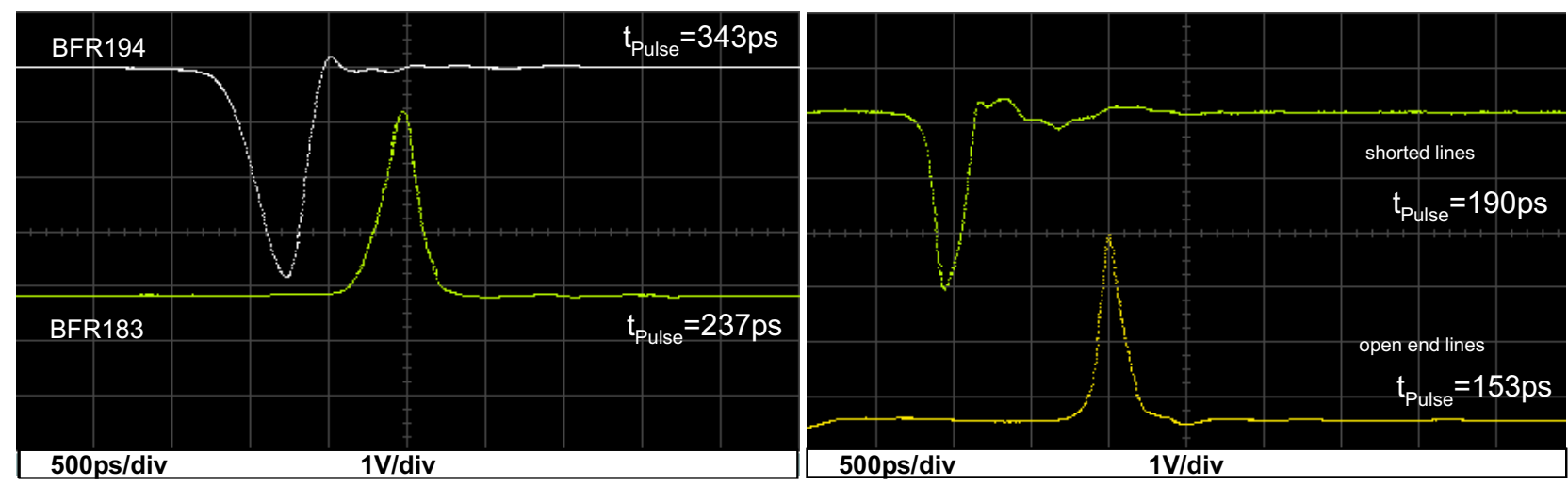

Fig. 9. Left: different pulse polarities caused by different transistor technologies (npn and pnp), right: changing the pulse polarity by different coupled line structures.

\section{Conclusion}

A robust comb generator based on fast rf-transistors has been presented. The complexity of the circuit design shown in Fig. 10 is relatively low, and since it uses standard components it is also quite cost effective.

The main characteristics are the flexibility in choosing the pulse length, the pulse amplitude and the choice of the pulse polarity. Each of the two complementary structures, one based on a npn-transistor, the other on a pnp-transistor, can be modified by the use of various differentiating networks to modify the pulse shape in detail. The maximum output peak pulse power is about $1 \mathrm{~W}$ with $7 \mathrm{~V}$ at $50 \Omega$ and a pulse duration between $90 \mathrm{ps}$ up to $800 \mathrm{ps}$ can be obtained. This results in baseband signals with a high harmonic content up to $10 \mathrm{GHz}$.

Fields of application for such comb generators are for example the time domain reflectometry, distance to fault measurements on transmission-lines and level gauging measurements.

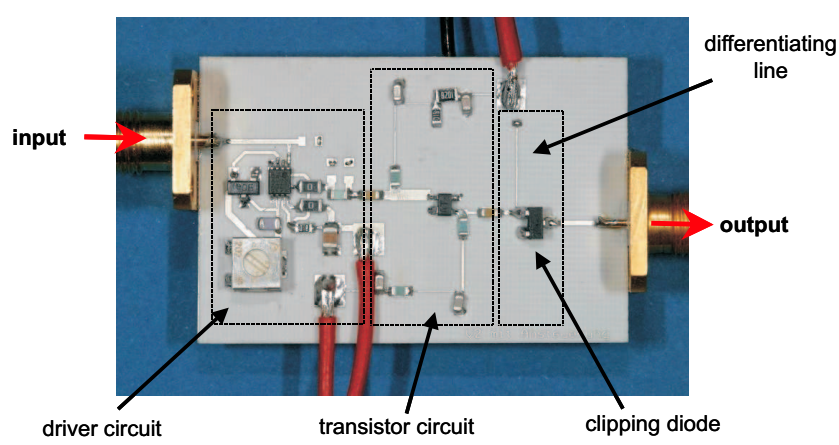

Fig. 10. Pulse generator circuit with shorted branch line and clipping diode. 


\section{References}

Hewlett Packard: Application Notes AN918, Pulse and Waveform Generation with Step Recovery Diodes.

Hewlett Packard: Application Notes AN920, Step Recovery Diodes, 1988

Hoffmann, K.: Modelle und Schaltungen, Oldenbourg, 3. Auflage, 1996.

Malherbe, J. A. G.: Microwave Transmission Line Filters, Artech House, 1979.
Matsumoto, A.: Microwave Filters and Circuits, Academic Press, 1970.

Paul, R.: Transistoren, Vieweg, 1965.

Schiek, B.: Grundlagen der Hochfrequenz-Messtechnik, Springer Verlag, 1999.

Schrenk, H.: Bipolare Transistoren, Springer Verlag, 1978.

Smith, P. W.: Transient Electronics, Wiley \& Sons Ltd, 2002.

Tietze, U. and Schenk, Ch.: Halbleiter Schaltungstechnik, 10. Auflage, Springer Verlag, 1993. 